\title{
Eigenmodes of Decay and Discrete Fragmentation Processes
}

\author{
B.G.Giraud and R.Peschanski \\ Service Physique Théorique, DSM-CE Saclay, F91191 Gif/Yvette, France \\ and \\ Wei-hsing Ma \\ Institute of High Energy Physics, Academia Sinica, 100039 Beijing, China
}

\begin{abstract}
Linear rate equations are used to describe the cascading decay of an initial heavy cluster into fragments. This representation is based upon a triangular matrix of transition rates. We expand the state vector of mass multiplicities, which describes the process, into the biorthonormal basis of eigenmodes provided by the triangular matrix. When the transition rates have a scaling property in terms of mass ratios at binary fragmentation vertices, we obtain solvable models with explicit mathematical properties for the eigenmodes. A suitable continuous limit provides an interpolation between the solvable models. It gives a general relationship between the decay products and the elementary transition rates.
\end{abstract}

In a previous paper ${ }^{1)}$ we considered binary fragmentation processes where any fragment with mass number $k$ breaks into fragments with mass numbers $j$ and $k-j, j=$ $1,2 \ldots k-1$, with a probability $w_{j k}$ per unit of time. It was assumed that $w_{j k}$ was time independent. By definition, $w_{j k}=0$ if $j \geq k$ and $w_{j k}$ is symmetric if $j$ is replaced by $k-j$, naturally. (For technical reasons, the coefficient $w_{j, 2 j}$ is twice the actual transition rate.) Let $N_{j}(t)$ be the multiplicity of fragment $j$ at time $t$ in a process initiated from the decay of a cluster $A$, namely $N_{j}(0)=\delta_{j A}$. The model under study is described by the following set of linear, first order differential equations,

$$
\frac{d N_{j}}{d t}=-c_{j} N_{j}+\sum_{k=j+1}^{A} w_{j k} N_{k}, j=1, \ldots A, \quad c_{j}=\sum_{\ell=1}^{j-1} \frac{w_{\ell j}}{2}
$$

With components $N_{j}, j=1, \ldots A$, for a column vector $|\mathcal{N}\rangle$, the system, Eqs.(1), boils down to $d|\mathcal{N}>/ d t=\mathcal{W}| \mathcal{N}>$ with a triangular matrix $\mathcal{W}$. The solution of Eqs.(1) is 
obviously $|\mathcal{N}>=\exp (-t \mathcal{W})| \mathcal{N}_{0}>$, a sum of exponentials whose rates of decay in time are the trivial eigenvalues of the triangular $\mathcal{W}$, namely the diagonal matrix elements $-c_{\lambda}$. The purpose of the present letter is to take advantage of the expansion of the evolution on the biorthonormal basis of eigenmodes of $\mathcal{W}$,

$$
\left|\mathcal{N}>=\sum_{\lambda=1}^{A}\right| \lambda>\exp \left(-t c_{\lambda}\right)<\tilde{\lambda}\left|\mathcal{N}_{0}>=\sum_{\lambda=1}^{A}\right| \lambda>\exp \left(-c_{\lambda} t\right) Y_{A}^{\lambda},
$$

where $Y_{A}^{\lambda}$ is the last component of the bra eigenvector $<\tilde{\lambda} \mid$. We are specially interested in the analytical structure of the bra eigenvectors $<\tilde{\lambda} \mid$, because of the synthetic information carried by scalar products $M_{\lambda}=<\tilde{\lambda} \mid \mathcal{N}>$.

In our previous investigations ${ }^{1)}$ we found numerical evidence for specific exponents $q(\lambda)$ which govern a power-like increase of the components of the bra eigenvectors, $Y_{j}^{\lambda} \propto$ $j^{q(\lambda)}$. We took advantage of the fact that $\mathcal{W}$ has a fixed bra eigenvector $<\tilde{1} \mid$, whose components $Y_{j}^{1}=j, j=1, \ldots A$, express mass conservation. Indeed the total mass is $M_{1}=<\tilde{1} \mid \mathcal{N}>=\sum_{j=1}^{A} j N_{j}$, with $d M_{1} / d t=<\tilde{1}|\mathcal{W}| \mathcal{N}>=0$, since the first eigenvalue $-c_{1}$ identically vanishes. It was also convenient to define the "mass weighted multiplicity" (MWM) vector $U$ with components $U_{i}=i N_{i}$, whose evolution is governed by a matrix $\mathcal{V}$, with matrix elements $\mathcal{V}_{j k}=j \mathcal{W}_{j k} / k$, hence $d U / d t=\mathcal{V} U$. A numerical analysis of $U$ revealed scaling properties of the fragmentation process. The present paper attempts to give analytical proofs where only numerical evidence was previously found. In particular we are interested in those mathematical properties of the bra $<\tilde{\lambda} \mid$ eigenvectors which may enlighten the comprehension of the expansion, Eq.(2).

Our main tools in this analysis are the following equations for the components $Y_{i}^{\lambda}$ of the bra eigenvectors of $\mathcal{W}$,

$$
\sum_{i=\lambda}^{j-2} Y_{i}^{\lambda} w_{i j}+Y_{j-1}^{\lambda} w_{j-1, j}=\left(c_{j}-c_{\lambda}\right) Y_{j}^{\lambda}, \quad j \geq \lambda .
$$

Similar equations govern the components $X_{i}^{\lambda}$ of the ket eigenvectors, naturally. We keep in mind that $Y_{j}^{\lambda}=0$ if $j<\lambda$ and $X_{i}^{\lambda}=0$ if $i>\lambda$. A suitable (biortho)normalization results from the following boundary conditions,

$$
Y_{\lambda}^{\lambda}=X_{\lambda}^{\lambda}=1 .
$$


Note that the components of the biorthonormal eigenvectors of the matrix $\mathcal{V}$ which governs the MWM are easily deduced, as $\mathcal{Y}_{j}^{\lambda}=\lambda Y_{j}^{\lambda} / j$ and $\mathcal{X}_{\rangle}^{\lambda}=i X_{i}^{\lambda} / \lambda$.

It happens frequently that $c_{j}$ is a monotonically increasing function of $j$. The physical interpretation is that the heavier the system, the larger its total decay rate. Under such a condition, the coefficients $c_{j}-c_{\lambda}$ in the right-hand side of Eqs.(3) are always positive when $j>\lambda$. The left-hand sides of the same, Eqs.(3), depends only on positive coefficients $w_{i j}$ and components $Y_{i}^{\lambda}$ of rank $i$ lower than $j, \lambda \leq i \leq j-1$. It is easy to calculate $Y_{\lambda+1}^{\lambda}$ from $Y_{\lambda}^{\lambda}$ alone, which was chosen to be positive from the normalization $Y_{\lambda}^{\lambda}=1$. Thus $Y_{\lambda+1}^{\lambda}$ is positive. A trivial recursive argument then shows that all non vanishing components $Y_{j}^{\lambda}$ are positive. ${ }^{1}$

In the same way as in our earlier ${ }^{1)}$ studies we first select values of $w_{i j}$ which express scaling properties at vertices of binary fragmentation. For this, the family of models we consider $\left.{ }^{2}\right)$ uses the form $w_{i j}=[f(i / j)+f(1-i / j)] j^{-a}$, and is parametrized by a "splitting" function $f$ and an overall exponent $a$. For most of the present analytical study, we restrict $a$ to the value $a=1$, because it allows a convenient continuous limit, valid for finite values of $\lambda$ and large sizes $A$ of the matrix. The value $a=1$ is for instance the proper exponent for QCD fragmentation ${ }^{3,4}$ ) when we consider energy-momentum fragmentation rather than mass fragmentation.

A simplified, albeit generic form of $f$ is parametrized by a second exponent, $b$, in the form

$$
w_{i j}=\left[\left(\frac{i}{j}\right)^{-b}+\left(1-\frac{i}{j}\right)^{-b}\right] j^{-a} .
$$

The variable $x=i / j$ (with its mandatory symmetry complement $1-x$ ) is obviously the scaling mass ratio at the fragmentation vertex. We note that the cases $a=1, b=0$ and $a=1, b=-1$ generate the same eigenvectors, since the corresponding matrices are strictly proportional, namely $\left.w_{i j}\right|_{b=0}=\left.2 w_{i j}\right|_{b=-1}=2 / j$.

1 As a consequence of the positivity of the bras and of biorthogonality, the ket eigenvectors must show highly oscillatory components. This demands a further, specific study. 
For $a=1, b=-1$, the simple nature of $w_{i j}$, namely $w_{i j}=1 / j$, reduces Eqs.(3) into

$$
\frac{j-1}{j}\left(c_{j-1}-c_{\lambda}\right) Y_{j-1}^{\lambda}+\frac{Y_{j-1}^{\lambda}}{j}=\left(c_{j}-c_{\lambda}\right) Y_{j}^{\lambda} .
$$

Hence a recursion relation for the bra eigenvector components is found,

$$
\frac{Y_{i}^{\lambda}}{Y_{i-1}^{\lambda}}=\frac{i+\lambda-1}{i-\lambda}
$$

and accordingly the bra eigenvector components are given by the formula,

$$
Y_{i}^{\lambda}=\frac{(i+\lambda-1) !}{(i-\lambda) !(2 \lambda-1) !} \text {. }
$$

For large values of $j$, the recursion relation expands in powers of $1 / j$, when $j$ is large, according to

$$
\frac{Y_{i+1}^{\lambda}}{Y_{i}^{\lambda}}=1+\frac{2 \lambda-1}{j}+\mathcal{O}\left(1 / j^{2}\right),
$$

which means that $Y_{i}^{\lambda} \propto i^{2 \lambda-1}$. This proves analytically that the scalar products $<\tilde{\lambda} \mid \mathcal{N}>=$ $\sum_{i=\lambda}^{A} Y_{i}^{\lambda} N_{i}$ of the bra eigenvectors with $\mathcal{N}$ express moments, of order $q(\lambda)=2 \lambda-1$, of the multiplicity distributions, restricted to fragments at least as heavy as $\lambda$. This justifies analytically, for $a=1$ and $b=-1,0$, the numerical evidence obtained in our previous paper $^{1)}$.

For $a=1, b=1$ the rates become $w_{i j}=j /[i(j-i)]$. The matrix $\mathcal{V}$ is simpler than $\mathcal{W}$, since then $\mathcal{V}_{i j}=1 /(j-i)$ in the upper-right triangle, which does not vanish. The diagonal is unchanged, and so is, naturally, the lower-left, vanishing triangle. A straightforward but slightly tedious argument shows that

$$
\mathcal{Y}_{j}^{\lambda}=\lambda Y_{j}^{\lambda} / j=\frac{(j-1) !}{(j-\lambda) !(\lambda-1) !} .
$$

One deduces from Eq.(9) that

$$
\frac{Y_{j+1}^{\lambda}}{Y_{j}^{\lambda}}=\frac{(j+1)}{(j+1-\lambda)}=1+\frac{\lambda}{j}+\mathcal{O}\left(1 / j^{2}\right),
$$

hence now $q(\lambda)=\lambda$. This makes a second case where the power law, $Y_{j}^{\lambda} \propto j^{q(\lambda)}$, can be proved analytically. 
If $a$ is frozen to the value $a=1$, it is tempting to interpolate between the behavior $q(\lambda)=2 \lambda-1$ found for $b=-1$ and that one, $q(\lambda)=\lambda$, valid for $b=1$. Because of the identity of eigenvectors for the $b=-1$ and $b=0$ cases, a parabolic interpolation

$$
q_{\text {int }}(\lambda)=\frac{\lambda\left(17-4 b^{2}\right)+4 b^{\prime 2}-9}{8}, \quad b^{\prime} \equiv b+\frac{1}{2}
$$

is found.

This interpolation turns out to be consistent with the continuous limit condition considered earlier, see Eq.(9) in reference ${ }^{1)}$,

$$
\begin{aligned}
c_{\lambda} & =\frac{\lambda^{b-1}}{2} \sum_{i=1}^{\lambda-1}\left[i^{-b}+(\lambda-i)^{-b}\right]=\lambda^{b-1} \sum_{i=1}^{\lambda-1} i^{-b} \\
& =\frac{1}{2} \int_{0}^{1} d x\left[1-x^{q}-(1-x)^{q}\right]\left[x^{-b}+(1-x)^{-b}\right]=\int_{0}^{1} d x\left[1-x^{q}-(1-x)^{q}\right] x^{-b},
\end{aligned}
$$

where we take advantage of the symmetry $w_{i j}=w_{j-i, j}$. We can use this continuous limit, Eq.(12), to estimate $q$ as a function of $\lambda$ when $c_{\lambda}$ is known, whether analytically or numerically. The identity of the cases $b=0$ and $b=-1$ for Eq.(12) is transparent. Also, for any value of $b$, the pair $\lambda=1, q=1$ solves obviously Eq.(12). Then, for $b=0$, one gets at once from Eq.(12) the condition $(\lambda-1) / \lambda=1-2 /(q+1)$, hence $q(\lambda)=2 \lambda-1$, in agreement with Eq.(8). Furthermore for $b=1$, with the ansatz $q(\lambda)=\lambda$, the recursive relation, derived from Eq.(12),

$$
c_{\lambda+1}-c_{\lambda}=\frac{1}{\lambda}=\int_{0}^{1} d x \frac{x^{\lambda}+(1-x)^{\lambda}-x^{\lambda+1}-(1-x)^{\lambda+1}}{x}
$$

is easy to verify.

We show on Fig. 1 the plots (full lines) of the values of $q(2), q(5)$ and $q(8)$, respectively, obtained numerically from Eq.(12) for $-1.5 \lesssim b \lesssim 1.5$. The same Fig. 1 shows (dotted lines) the corresponding interpolation predictions $q_{\text {int }}(\lambda)$ from Eq.(11). As long as $-1.2 \lesssim b \lesssim$ 1.2, the continuous limit results and the parabolic interpolations do not differ by more than $4 \%$. Significant deviations between such estimates occur, however, when $1.2 \lesssim|b|$. It may be pointed out that the continuous limit estimates are expected to be more rigorous. 
Finally on Fig.2 we show, for e.g. $b=-0.5, b=0.5$ and $b=1.5$, that, when provided by Eq.(12), the behavior of $q$ as a function of $\lambda$ remains essentially linear. Namely a parametrization $q(\lambda) \simeq \alpha(b) \lambda+\beta(b)$ is reasonable, despite the fact that $\alpha(b)$ and $\beta(b)$ are more complicated than the quadratic forms present in Eq.(11).

In conclusion, there is a firm ground for the validity of the power law which governs the bra eigenvector components and the corresponding interpretation in terms of moments of the multiplicity vector $\mathcal{N}$. The apparent simplicity of the empirical law $q(\lambda) \simeq \alpha(b) \lambda+$ $\beta(b)$, see for instance Eq.(11), expresses a non trivial relationship between very different quantities, namely experimentally observable moments on one hand and, on the other hand, much less observable parameters $b$ of the microscopic mechanism of binary fragmentation. Acknowledgments : One of the authors (W-X Ma) thanks the French Atomic Energy Commission for a stay at Service de Physique Théorique, Saclay, where this work was performed.

\section{References}

1) B.G. Giraud and R. Peschanski, Phys.Lett.B315,452(1993)

2) Z. Cheng and S. Redner, J.Phys.A:Math.Gen.23,1233(1990); E.D. Mc Grady and Robert M. Ziff, Phys.Rev.Lett.58,892(1987)

3) G. Altarelli and G. Parisi, Nucl.Phys.126,297(1977); V.N. Gribov and L.N. Lipatov, Sov.Journ.Nucl.Phys.15,438,675(1972); For a review and references, see Y.L. Dokshitzer, V.A. Khoze, A.H. Mueller and S.I. Troyan, Basics of perturbative QCD (J. Tran Than Van ed. Editions Frontières, France, 1991)

4) P. Cvitanovic, P. Hoyer and K. Zalewski, Nucl.Phys.B176,429(1980)

\section{Figure Captions}

Fig.1 : Behavior of the "eigenexponents" $q(\lambda)$ for the 2nd, 5th and 8th bra eigenvectors, as functions of the parameter $b$ (model with scaling properties for fragmentation vertices). Full lines: continuous limit predictions. Dotted lines: quadratic interpolations between the exact values, known analytically for $b=-1,0,1$.

Fig.2 : Strongly linear behavior of $q(\lambda)$ as a function of $\lambda$, for different values of $b$. Full lines are drawn to guide the eye between integer values of $\lambda$. 
This figure "fig1-1.png" is available in "png" format from: http://arxiv.org/ps/nucl-th/9412026v1 
This figure "fig1-2.png" is available in "png" format from: http://arxiv.org/ps/nucl-th/9412026v1 